\title{
Defiant Denial is Self-Defeating
}

\author{
E.J. Wagenmakers \\ University of Amsterdam
}

Address for correspondence:

EJ.Wagenmakers@gmail.com

Department of Psychological Methods, room G 0.29

University of Amsterdam, Nieuwe Achtergracht 129B

Letter: PO Box 15906, 1001 NK Amsterdam

Parcel: Valckenierstraat 59, 1018 XE Amsterdam 
In what is arguably one of the most iconic scenes of the comedy film "The Naked Gun", police lieutenant Frank Drebin confronts his antagonist Vincent Ludwig on top of the stands in a baseball stadium, shooting him in the neck with a cufflink dart. As Ludwig grabs his neck and starts to groan, the lady next to Drebin says “you killed him!”. Drebin replies, "No, no, he's just stunned. He'll be alright in a couple of minutes." But Ludwig stumbles and trips over the perimeter wall, falling from great height unto the ground below. Subsequently, Ludwig's body is run over by a bus, flattened by a steamroller, and finally trampled by the USC Trojans marching band playing "Louie Louie". On seeing the carnage unfold, Drebin's colleague Ed Hocken breaks down and says "Oh Frank, it's horrible, it's so horrible...my father went the same way."

In what follows I will use this scene to draw an admittedly tortured analogy with social psychology and the demise of "social priming" research. In this analogy, Frank Drebin represents Kahneman, Vincent Ludwig represents the field of social psychology, the lady accusing Drebin represents Sherman and Rivers, and Ed Hocken represents the academics who are not working in social psychology. Each of the sections below concentrates on one of the actors. Before proceeding, I should stress that I agree with Sherman and Rivers (this issue) on two important points. First, I agree that the sample sizes typically used in between-subjects social priming research have generally been much too low, and that the resulting experiments were severely underpowered (cf. Harris et al., 2013; Pashler, Coburn, \& Harris, 2012, who also argued that it is remarkable that the reported effect sizes for social priming are substantially larger than the effect sizes for perceptual priming). Second, I wholeheartedly support the call for more mathematical modeling in social psychology (e.g., Calanchini et al, 2018; Calanchini, Lai, \& Klauer, in press; Klauer et al., 2007). 


\section{DEFIANT DENIAL IS SELF-DEFEATING}

Also, I should state up front that the field of social psychology deserves praise for how it has handled the "crisis of confidence" (Pashler \& Wagenmakers, 2012). Many recent trail-blazing methodological reforms have been initiated or promoted by social psychologists while disregarding the remonstrations and ridicule from conservative senior peers. From a methodological perspective, social psychology has effectively reinvented itself within a single decade. Because of this metamorphosis, social psychology now sets an example for other fields, many of which are only starting to wake up from blissful dreams to face the inconvenient reality that experimenters are often mere flotsam in a sea of hindsight bias and motivated reasoning. In the words of Barber (1976, p. 20):

"When not planned beforehand, data analysis can approximate a projective technique, such as the Rorschach, because the investigator can project on the data his own expectancies, desires, or biases and can pull out of the data almost any 'finding' he may desire."

Any discussion of the crisis of confidence that omits Barber's "investigator data analysis effects" is seriously incomplete. We are now ready to explore our analogy with the Naked Gun scene.

\section{The Role of Frank Drebin/Kahneman}

Frank Drebin did not intend to kill Vincent Ludwig any more than Daniel Kahneman intended to damage the field of social psychology or the subfield of social priming. As is 
abundantly clear from his book "Thinking Fast Thinking Slow", Kahneman was firmly convinced of the veracity of the social priming findings:

"When I describe priming studies to audiences, the reaction is often disbelief (...) The idea you should focus on, however, is that disbelief is not an option. The results are not made up, nor are they statistical flukes. You have no choice but to accept that the major conclusions of these studies are true. More important, you must accept that they are true about you.” (Kahneman, 2011, pp. 56-57).

In his "train wreck" email, Kahneman expressed the hope that the priming protagonists would coordinate their efforts and engage in a systematic "daisy chain" replication effort in order to dispel any doubt about the presence of these priming effects. His letter was not motivated by scepticism, nor was it a devious plan to trick the protagonists: it was a sincere attempt to gather the kind of empirical evidence that would convince the skeptics that they were wrong. In the words of Kahneman:

"I believe that you should collectively do something about this mess. To deal effectively with the doubts you should acknowledge their existence and confront them straight on, because a posture of defiant denial is self-defeating. Specifically, I believe that you should have an association, with a board that might include prominent social psychologists from other field [sic]. The first mission of the board would be to organize an effort to examine the replicability of priming results, following a protocol that avoids 


\section{DEFIANT DENIAL IS SELF-DEFEATING}

the questions that have been raised and guarantees credibility among colleagues outside the field." (Kahneman, 2012)

But Kahneman's proposal was ignored, fueling suspicion from outsiders that the priming protagonists were themselves doubtful that their findings would replicate successfully.

\section{The Role of the Worried Lady/Sherman and Rivers}

Throughout their article, Sherman and Rivers take on a posture of defiant denial. They go to great efforts to explain that "social priming" is a misnomer (do we care what it is called?), and that some priming effects in social psychology do appear to replicate (one swallow does not a summer make; more about replication in social psychology shortly). They also identify a simple statistical reason -lack of power in between-subjects designs- as the main culprit for the ubiquitous problem of non-replicability.

Similar to the lady who accused Frank Drebin of killing Vincent Ludwig, Sherman and Rivers accuse Kahneman of plotting to damage social psychology when they state that "Kahneman threw the whole field of social psychology under the bus, not only with respect to reproducibility, but also with insinuations of outright fraud." As outlined in the previous section, this accusation appears entirely unfounded. Inadvertently, this false accusation illustrates the kind of scientific culture that fosters the routine production of non-replicable results, that is, an in-group culture in which even preposterous claims go unchallenged for fear of being ostracized. Ostracizing Kahneman is not an effective strategy, of course, but it may make early career researchers think twice before suggesting to their peers that key results in the field warrant replication. 


\section{The Role of Vincent Ludwig/Social Psychology}

If it were just the case that social priming did not replicate, the field of social psychology would be stunned, but not fall from great height to be trampled on by the USC Trojans marching band. However, the problems for social psychology ran much deeper than social priming. The Bem saga is problematic, not because the flagship journal for social psychology (i.e., the Journal of Personality and Social Psychology or JPSP) published an article about extra-sensory perception (Bem, 2011), but because it showed one could publish nonsense in JPSP using common tools of the trade. In their reply "Why psychologists must change the way they analyze their data", Wagenmakers et al. (2011) argued that "The field of psychology currently uses methodological and statistical strategies that are too weak, too malleable, and offer far too many opportunities for researchers to befuddle themselves and their peers.” (p. 426), and that

"Instead of presenting exploratory findings as confirmatory, one should ideally use a two-step procedure. First, in the absence of strong theory, one can explore the data until one discovers an interesting new hypothesis. But this phase of exploration and discovery needs to be followed by a second phase, one in which the new hypothesis is tested against new data in a confirmatory fashion. This is particularly important if one wants to convince a skeptical audience of a controversial claim: After all, confirmatory studies are much more compelling than exploratory studies. Hence, explorative elements in the research program should be explicitly mentioned, and statistical results should be adjusted accordingly.” (p. 427). 
The case of Stapel was likewise problematic, not because Stapel was a social psychologist who faked data in 55 publications, but because the fraud could go undetected for many years - Stapel's fraud was not detected by a failure to replicate, it was detected by his $\mathrm{PhD}$ students. This makes Bem and Stapel symptoms of a systemic dysfunction (e.g., the Levelt-Noort-Drenth report, 2012; Wagenmakers et al., 2011).

But the true downfall for social psychology happened when it turned out that replication efforts almost always yielded disappointing results. Here I will mention two prominent examples (cf. Wagenmakers et al., 2017). First, Figure 1 shows the results for the Replication Project: Psychology (RP:P; The Open Science Collaboration, 2015) with the JPSP studies highlighted. The RP:P article mentions that "Average effect size in JPSP did not differ from $0(.042 ; z=.71$, $p=.48)$ " (Open Science Collaboration, 2015, p. 45). The leftmost green dot reflects a significant effect because it was a psychometric study with 230,047 participants. As mentioned in Wagenmakers et al. (2017):

"Figure 1 paints a bleak picture: all but one of the replicated effect sizes lie below the diagonal, meaning that they are smaller than those originally published in JPSP; some replicated effect sizes are negative, whereas others are so small that - even if these effects were real- it would take much larger sample sizes for them to be meaningfully studied (e.g., Button et al., 2013); finally, 6 out of 31 replication studies were statistically significant, compared to 30 out of 31 for the original experiments published in JPSP." 


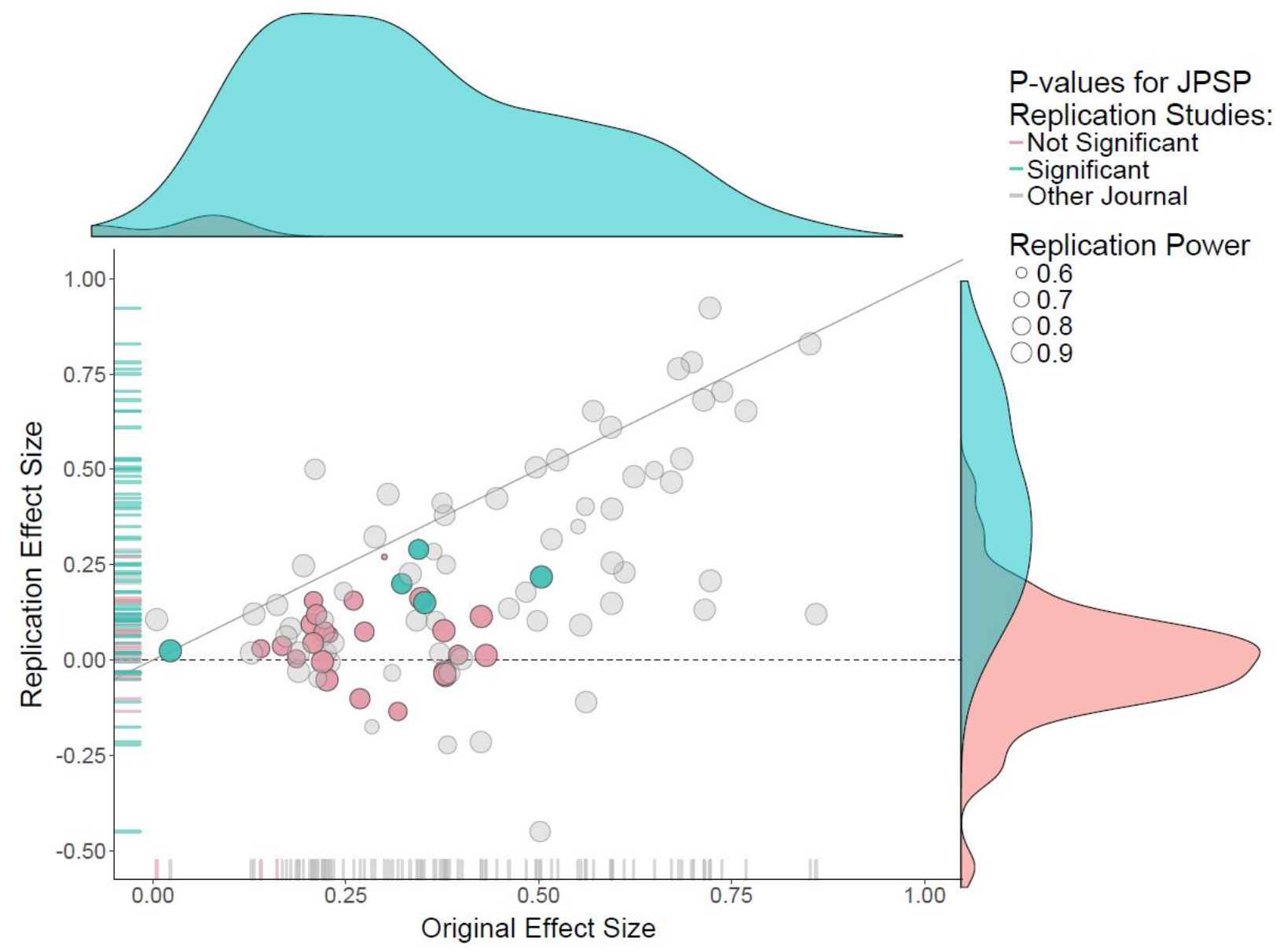

Figure 1. "Results from the replication attempts conducted as part of the Replication Project: Psychology. The results for the replication attempts of JPSP articles are highlighted. Courtesy of Fred Hasselman, this graph is an edited version of the one presented in the Open Science Collaboration (2015) . The associated information is available on the Open Science Framework at https://cos.io/pr/2015-08-27/." Figure and associated text from Wagenmakers et al. (2017).

Second, Figure 2 shows the result from a Bayesian reanalysis of the special issue "Replications of important results in social psychology" (Nosek \& Lakens, 2013, 2014). 


\section{DEFIANT DENIAL IS SELF-DEFEATING}

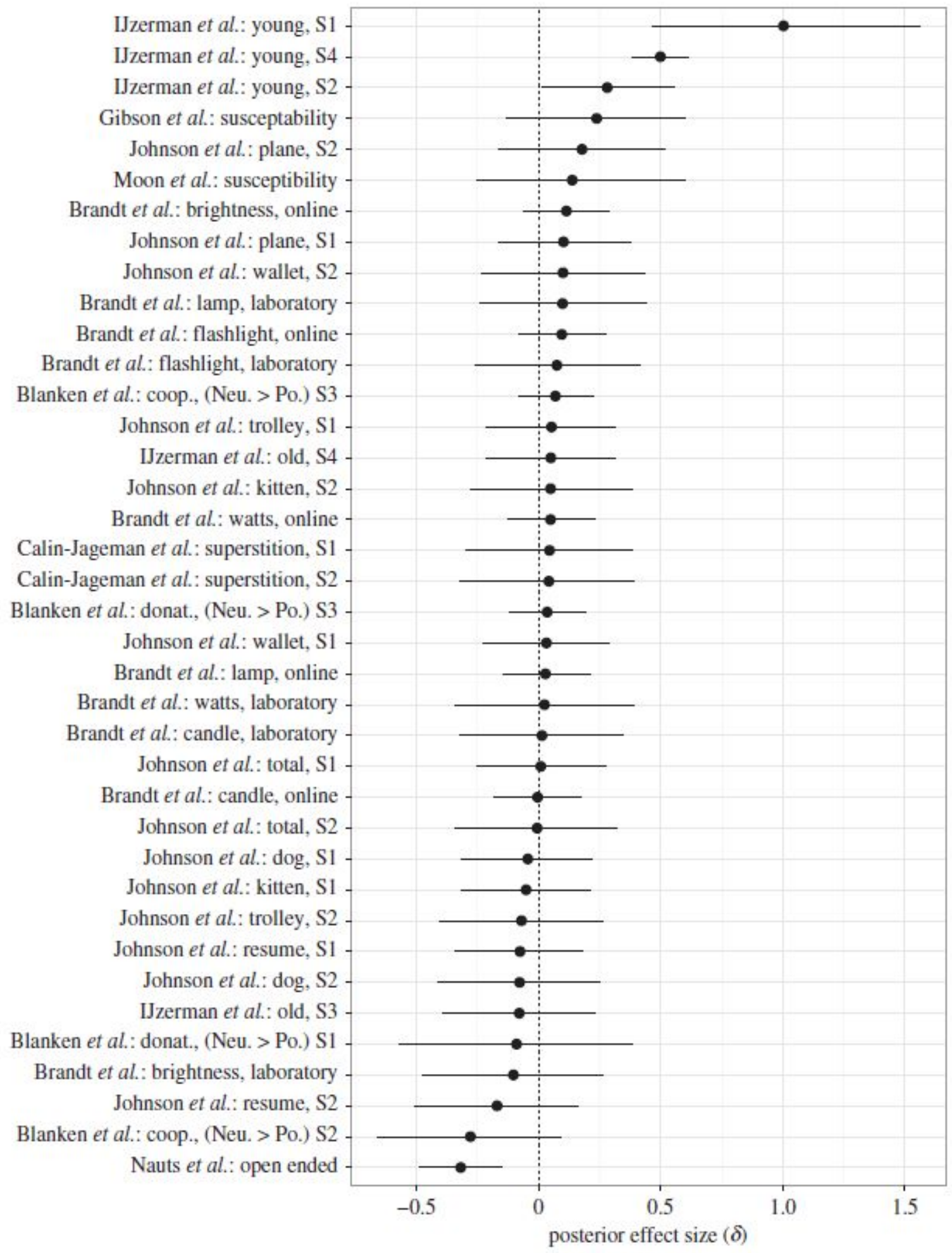

Figure 2. Bayesian parameter estimation results for each of 38 experiments from the Nosek \& Lakens (2014) Social Psychology special issue that used $t$-tests. Positive effect sizes are consistent with the original study. Figure available at https:/flic.kr/p/FQJrUr, under CC license https://creativecommons.org/licenses/by/2.0/. For details see Marsman et al. (2017). 
It is immediately clear that the overall replication success is abysmal. This occurred despite the fact that considerable care was taken to design the replication studies with high power, in consultation with the original authors, and as a Registered Report (e.g., Chambers, 2013, 2017).

A lack of power is an unlikely root cause for the replicability woes in social priming or social psychology more broadly. The deeper problem I believe is that implausible findings are being probed in an exploratory fashion by means of a trigger-happy methodology (Benjamin et al., 2018) that is only appropriate for purely confirmatory tests; moreover, data analysis is conducted by researchers fully invested in the outcome, without any accountability, with no protection from hindsight bias, and with every incentive to obtain a "significant" result. One does not need to be a social psychologist to realize that this scenario is not conducive to the production of reliable knowledge.

\section{The Role of Ed Hocken/The Academic Outgroup}

When confronted with the results from the earlier section (and the dismal replication success in social psychology across the board) academic outsiders may not know whether to laugh or to weep. Ed Hocken wept (“horrible!"), but Sherman and Rivers suggest that "The schadenfreude following replication failures of the research was (and is) thick on social media and in conference hotel bars." Sherman and Rivers attribute this to "long-standing grievances about the attention paid to some of the social priming effects". This is a euphemism; the grievances were caused in part by the fact that established social psychologists were able to publish shoddy and implausible work in prestigious outlets on a regular basis. This is indicative of a systemic problem. Discontent was already brewing within social psychology itself, and the failures to replicate the social priming findings were merely the spark that lit the fuse. It may be 


\section{DEFIANT DENIAL IS SELF-DEFEATING}

motivating to see your colleagues soar academically, but not when you know their wings are made of wax.

Sherman and Rivers worry about the public perception of the field of social psychology and its consequences. They state:

\footnotetext{
"Administrations, departments, and individual scientists who knew little or nothing about social psychology, not to mention priming research in social psychology, suddenly had strong doubts about the field and its researchers. It is difficult to quantify the damage, but few would argue that the episode didn't impact publishing, grant funding, and employment for social psychologists."
}

But this is a matter of academic crime and punishment. If the published literature of a field proves to be a highly unreliable guide to knowledge, then the reputation of academics working in this field will naturally take a hit, with ensuing consequences for publishing and grant funding. Vincent Ludwig got what he deserved, and so did the field of social psychology, at least to some degree.

However, not all is doom and gloom. As mentioned in the introduction, many social psychologists have made it their mission to help social psychology reinvent itself by raising the methodological standards in the field. The submission guidelines for JPSP now stress the importance of open data, open materials, preregistration, and replication. Social psychologists have played and continue to play a prominent role in various Open Science initiatives and it is no exaggeration to say that the field has undergone a scientific revolution (Spellman, 2015; Spellman, Gilbert, \& Corker, 2018). 


\section{DEFIANT DENIAL IS SELF-DEFEATING}

Thus, I believe that, rather unlike the ending of the Naked Gun, social psychology is in the process of rising like a phoenix from the flames. And this is necessary, not just because early career researchers wish to build their careers on a solid empirical foundation, but also because, at its core, social psychology concerns some of the most interesting questions about human nature. There is constructive progress to be made here, and in many ways there has never been a better time to pursue a career in social psychology than right now. 


\section{References}

Barber, T. X. (1976). Pitfalls in human research: Ten pivotal points. New York: Pergamon Press Inc.

Bem, D. J. (2011). Feeling the future: Experimental evidence for anomalous retroactive influences on cognition and affect. Journal of Personality and Social Psychology, $100,407-425$.

Benjamin, D. J., Berger, J. O., Johannesson, M., Nosek, B. A., Wagenmakers, E.-J. ... \& Johnson, V. E. (2018). Redefine statistical significance. Nature Human Behaviour, 2, 6-10.

Button, K. S., Ioannidis, J. P. A., Mokrysz, C., Nosek, B. A., Flint, J., Robinson, E. S. J., \& Munafò, M. R. (2013). Power failure: Why small sample size undermines the reliability of neuroscience. Nature Reviews Neuroscience, 14, 1-12.

Calanchini, J., Rivers, A. M., Klauer, K. C., \& Sherman, J. W. (2018). Multinomial processing trees as theoretical bridges between cognitive and social psychology. Psychology of Learning and Motivation, 69, 39-65.

Calanchini, J., Lai, C. K., \& Klauer, K. C. (in press). Reducing implicit racial preferences: III. A process-level examination of changes in implicit preferences. Journal of Personality and Social Psychology.

Chambers, C. D. (2013). Registered Reports: A new publishing initiative at Cortex. Cortex, 49, 609-610.

Chambers, C. D. (2017). The seven deadly sins of psychology: A manifesto for reforming the culture of scientific practice. Princeton: Princeton University Press.

Harris, C. R., Coburn, N., Rohrer, D., \& Pashler, H. (2013) Two failures to replicate high-performance-goal priming effects. PLOS ONE 8: e72467.

Kahneman, D. (2011). Thinking, fast and slow. London: Allen Lane.

Kahneman, D. (2012). A proposal to deal with questions about priming effects. 
Klauer, K. C., Voss, A., Schmitz, F., Teige-Mocigemba, S. (2007). Process components of the implicit association test: A diffusion-model analysis. Journal of Personality and Social Psychology, 93, 353-368.

Levelt Committee, Noort Committee, \& Drenth Committee (2012). Flawed science: The fraudulent research practices of social psychologist Diederik Stapel.

Marsman, M., Schönbrodt, F., Morey, R. D., Yao, Y., Gelman, A., \& Wagenmakers, E.-J. (2017). A Bayesian bird's eye view of "Replications of Important Results in Social Psychology". Royal Society Open Science, 4: 160426.

Nosek, B. A., \& Lakens, D. (2013). Call for proposals: Special issue of Social Psychology on "Replications of important results in social psychology". Social Psychology, 44, 59-60.

Nosek, B. A., \& Lakens, D. (2014). Registered reports: A method to increase the credibility of published results. Social Psychology, 45, 137-141.

Pashler. H., Coburn, N., \& Harris, C. R. (2012). Priming of social distance? Failure to replicate effects on social and food judgments. PLoS ONE, 7: e42510.

Pashler, H., \& Wagenmakers, E.-J. (2012). Editors' introduction to the special section on replicability in psychological science: A crisis of confidence? Perspectives on Psychological Science, 7, 528-530.

Sherman, J. W., \& Rivers, A. M. (this issue). There's nothing social about social priming: Derailing the "train wreck". Psychological Inquiry.

Spellman, B. A. (2015). A short (personal) future history of revolution 2.0. Perspectives on Psychological Science, 10, 886-899.

Spellman, B. A., Gilbert, E. A., \& Corker, K. S. (2018). Open science. In J. Wixted \& E.-J. Wagenmakers (Eds.), Stevens' handbook of experimental psychology and cognitive neuroscience (4th ed.), Volume 5: Methodology (pp. 297-322). New York, NY: Wiley.

The Open Science Collaboration (2015). Estimating the reproducibility of psychological science. Science, 349, aac4716.

Wagenmakers, E.-J., Wetzels, R., Borsboom, D., \& van der Maas, H. L. J. (2011). Why psychologists must change the way they analyze their data: The case of psi: Comment on Bem (2011). Journal of Personality and Social Psychology, 100, 426-432. 


\section{DEFIANT DENIAL IS SELF-DEFEATING}

Wagenmakers, E.-J., Marsman, M., van den Bergh, D., Chambers, C., Pashler, H., De Ruiter, J., Fischer, A., Giner-Sorolla, R., Inzlicht, M., Jonas, K., Cesario, J., Borsboom, D., van der Maas, M., Harris, C., Freitas, A., Vazire, S., Gervais, W., Milyavskaya, M., Dunn, E., McCullough, M., Inbar, Y., Dijksterhuis, A., Doosje, B., Rimé, B., van Beest, I., Urry, H., \& Tullett, A. (2017). Suggestions to advance your mission: An open letter to Dr. Shinobu Kitayama, editor of JPSP:ASC. PsyArXiv: https://psyarxiv.com/39ugj. 